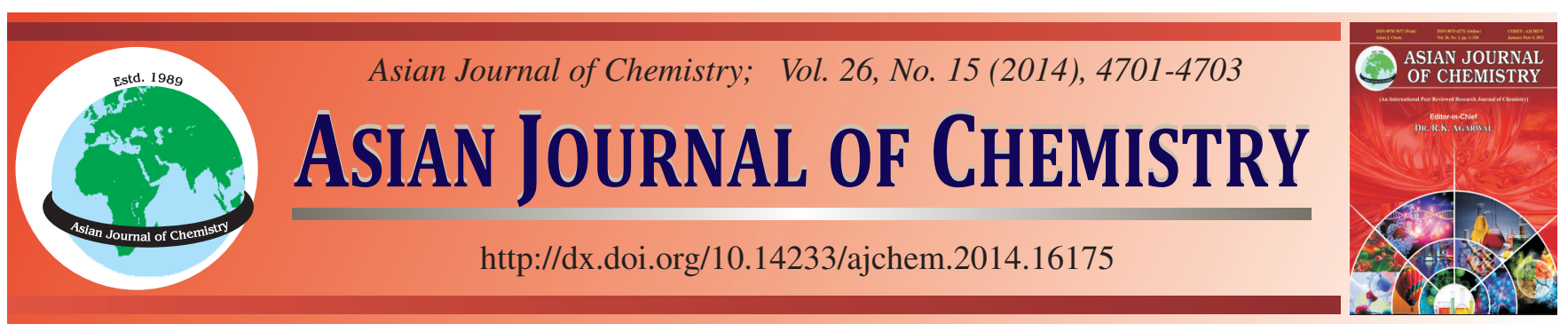

\title{
Synthesis of Brominated Quercetin Derivatives Using Distinct Brominating Systems
}

\author{
Mei Peng ${ }^{1}$, Fengxian Liu ${ }^{1}$, Xin Feng $^{1}$, Fan Yang $^{2}$ and Xiaoping Yang ${ }^{1, *}$
}

${ }^{1}$ Department of Pharmacy, School of Medicine, Hunan Normal University, Changsha 410013, P.R. China

${ }^{2}$ State Key Laboratory of the Discovery and Development of Novel Pesticide, Shenyang Research Institute of Chemical Industry Co. Ltd., Shenyang 110021, P.R. China

*Corresponding author: Fax: +86 731 88912417; Tel: +86 731 88912400; E-mail: simonxyoung@yahoo.com

6,8-Dibromoquercetin derivatives were obtained through reaction of quercetin with $\mathrm{HBr}-\mathrm{H}_{2} \mathrm{O}_{2}$. On the other hand, quercetin reacted with bromine to synthesize poly brominated derivatives. The reaction procedures were monitored by either TLC or HPLC or both in some cases. The reaction products were further purified via either recrystallization or silica gel column chromatography. Then the chemical structures were further confirmed by ${ }^{1} \mathrm{H}$ NMR and LC-MS. Two new quercetin brominated derivatives, namely 6,8-dibromoquercetin and $6,8,2^{\prime}, 5^{\prime}, 6^{\prime}$-pentabrominated quercetin were obtained. Two distinct brominated approaches were studied to obtain two different quercetin brominated derivatives, 6,8-dibromoquercetin and 6,8,2',5',6'-pentabrominated quercetin with the yields of 80 and $70 \%$, respectively.

Keywords: Quercetin, Selectivity, Bromination.

\section{INTRODUCTION}

Quercetin is widely distributed in varying concentrations in vegetables and fruits ${ }^{1}$. It has extensive pharmacological effects, mainly concentrated on antitumor, antioxidation and antiinflammatory activity ${ }^{2,3}$. In the United States, quercetin has been approved as a non-prescription drug widely used to treat prostate cancer (http://baike.baidu.com/view/140548.htm). So far, people have done much researches to modify the chemical structure of quercetin for the purpose of obtaining more active compounds via etherification ${ }^{4}$, esterification ${ }^{5}$, Sydney Mannich reaction ${ }^{6}$ and metal complexes of quercetin ${ }^{7,8}$. However, the synthesis of quercetin brominated derivatives is rare to report ${ }^{9,10}$.

In general, chemical reagents commonly used for the bromination are hydrobromic acid, bromine and N-bromo succinimide. For flavonols, the reaction mechanism is that hydrogens on the benzene rings are replaced by bromine atom through electrophilic substitution reaction, forming brominate derivatives. However, the similarity of chemical activity of various hydrogens on the benzene rings may lead to a number of different derivatives, which are difficult to isolate and purified. Therefore, it is challenging to regional selectively control brominated levels with different number of hydrogen replacement on benzene ring. On the other hand, the hydroxyl of flavonols is also prone to cause side effects, resulting in decrease in yields.
Considering these factors, we have designed two different brominated systems: one of which had mild reaction conditions while the other was violent. We expected that the mild condition will result in low level of hydrogen replacement with mono brominated derivative while violent condition will produce high level of hydrogen replacement with poly brominated product. We tested the role of solvents, the temperature and time of reaction and developed two effective bromination methodologies. The structures of the compounds obtained have been confirmed by LC-MS and NMR spectra.

\section{EXPERIMENTAL}

6,8-Dibromoquercetin: To a suspension of quercetin $(1 \mathrm{~g})$ in $\mathrm{MeOH}(50 \mathrm{~mL}), 30 \% \mathrm{H}_{2} \mathrm{O}_{2}(3.5 \mathrm{~mL})$ was added dropwise at $0-5{ }^{\circ} \mathrm{C}$ and then $47 \% \mathrm{HBr}(1 \mathrm{~mL})$ was added dropwise for $10 \mathrm{~min}$. After being stirred at $0-5^{\circ} \mathrm{C}$ for $3 \mathrm{~h}, 1.5$ $\mathrm{mL} \mathrm{H}_{2} \mathrm{O}_{2}$ was dropped and $0.5 \mathrm{~mL} 47 \% \mathrm{HBr}$ was slowly added and further stirred for $2 \mathrm{~h}$. The reaction mixture obtained was poured into $\mathrm{H}_{2} \mathrm{O}(300 \mathrm{~mL})$ to quench. The precipitate was collected by suction, washed with $\mathrm{H}_{2} \mathrm{O}$ and dried. The precipitate was dissolved in ethyl acetate solvent and then extracted with ethyl acetate and water (1:2), dried over anhydrous magnesium sulfate, removed the solvents under rotor vacuum and then recrystallized from acetone-DMF. This gave $1.2 \mathrm{~g}$ of yellow acicular crystals red-brown solids with mp $250-252^{\circ} \mathrm{C}$. The yield of 6,8-dibromoquercetin was $80 \%$. 
$\mathbf{6 , 8}, 2^{\prime}, 5^{\prime}, 6^{\prime}$-Pentabrominated quercetin: A solution of $3 \mathrm{~g}(0.01 \mathrm{~mol})$ of quercetin in $100 \mathrm{~mL}$ of glacial acetic acid was treated dropwise with $4.3 \mathrm{~mL}(0.08 \mathrm{~mol})$ of bromine in $30 \mathrm{~mL}$ of glacial acetic acid. The reaction mixture was stirred at room temperature for $3 \mathrm{~h}$ and was then increased to $85^{\circ} \mathrm{C}$ in an oil bath and further stirred for $2 \mathrm{~h}$ and evaporated under vacuum. The residue was washed with distilled water to eliminate traces of acid and bromine and then extracted with ethyl acetate, evaporated under reduced pressure. This directly gave $5 \mathrm{~g}$ of red-brown solids with mp $260-262{ }^{\circ} \mathrm{C}$. The yield of $6,8,2^{\prime}, 5^{\prime}, 6^{\prime}$-pentabrominated quercetin was $70 \%$.

HPLC analysis was measured on a Waters e2695-2998. Melting points were determined on a Buchi (M-565) melting point apparatus and were uncorrected. NMR spectra were acquired on a Gemini $300 \mathrm{MHz}$ NMR spectrometer and were reported relative to tetramethylsilane as an internal standard, dimethylsulfoxide as solvent. LC-MS of compounds were recorded by Agilent 1100 Series LC/MSD Trap SL.

The samples dissolved in ethyl acetate was loaded into the TLC plate and then developed in ethyl acetate :methanol 30:1 solvent system. After the solvents reached the top of the TLC plate, the plate was taken out and dried. The TLC plate was coloured and then image was taken by Iphone camera. Lane 1: starting material quercetin, Lane 2: mixture of material and product, Lane 3: reaction product, lane 4:mixture of reaction product and crystalline product, lane 5:crystalline product (Fig. 1).

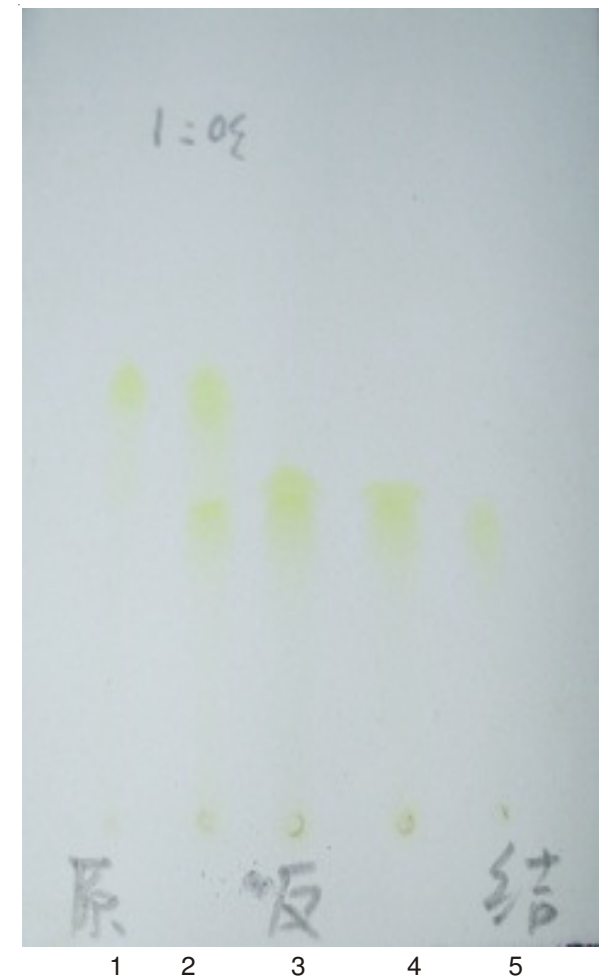

Fig. 1. TLC image of the reaction of quercetin with $\mathrm{HBr}-\mathrm{H}_{2} \mathrm{O}_{2}$ system

\section{RESULTS AND DISCUSSION}

Scheme-I: Hydrobromic acid in an oxidation system had a marked tendency to lead to 6,8-dibromoflavonols. Controlling the ratio of starting materials and reaction temperature is

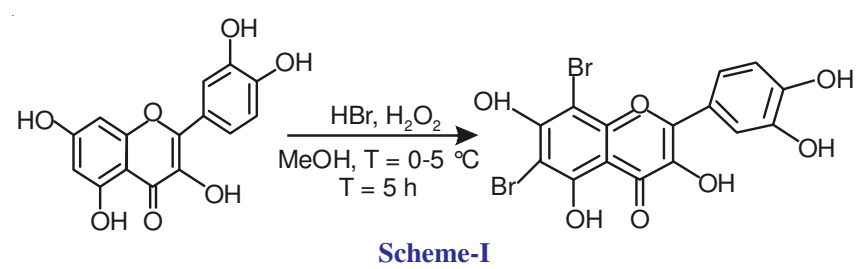

critical to obtain targeted product with high yield and good purity. Interestingly, we could not obtain high content of mono brominated quercetin -even under milder conditions including decreasing the temperature, lower levels of hydrobromic acid and shortening the reaction time. This indicates that two hydrogens at 6- and 8-positions are equivalently active and not discriminative under these conditions.

The NMR spectra of quercetin were characterized by two narrow doublets at approximately $\delta 6.20$ and $\delta 6.45$ for the 6- and 8-hydrogens, respectively. The NMR spectra for this product at mild conditions displayed neither hydrogen peaks near $\delta 6.20$ nor $\delta 6.45$, indicating that the product is 6.8 dibromoflavonol. Its mass spectra showed that its molecular mass was 460.7 and had the A, A +2 pattern in the correct ratio which is characteristic of fragments containing two bromine atoms.

We have investigated the effects of reaction temperature and molar ratio of quercetin and $\mathrm{HBr}$ on the components of product (Scheme-I). It is found that the reaction temperature played a major role in controlling the products. If the reaction temperature was controlled at $0-5{ }^{\circ} \mathrm{C}$, the product would be pure 6,8-dibromoquercetin with little by-product showed by TLC. However, if the reaction temperature was gradually increased, reaching at $20{ }^{\circ} \mathrm{C}$, the products would be a variety of different components, including a series of polybrominated quercetin, which were analyzed by HPLC and were found that it was difficult to separate the products. The ratio of starting materials is another critical factor to affect the products. Using the similar TLC and HPLC monitoring approaches, we studied the effect of the ratio of quercetin and $\mathrm{HBr}$ on distribution of products. We found that 1:1.5 ratio of quercetin: $\mathrm{HBr}$ gave best final target product 6,8-dibromoquercetin (Table-1). Therefore, to obtain high quality target product 6,8-dibromoquercetin, the optimized reaction conditions are reaction temperature: $0-5{ }^{\circ} \mathrm{C}$, ratio of starting materials: quercetin: $\mathrm{HBr}: \mathrm{H}_{2} \mathrm{O}_{2}=$ 1:1.5:1.5(g/mL/mL)(Table-1). To obtain a better quality of products, we investigated the purification processes under various solvent systems. It turned out that recrystallization using acetone with a few drops of DMF as a solvent system is the best.

TABLE-1

REACTION OF QUERCETIN WITH HBR IN THE FOLLOWING DIFFERENT CONDITIONS AND THE EXPLORATION RESULT

\begin{tabular}{ccc}
\hline Entry & $\begin{array}{c}\text { Ratio of material } \\
\text { (quercetin: } 47 \% \mathrm{HBr} \text { ) }\end{array}$ & $\begin{array}{c}\text { Reaction } \\
\text { temperature }\end{array}$ \\
\hline 1 & $1 \mathrm{~g}: 0.7 \mathrm{~mL}$ & $0-5{ }^{\circ} \mathrm{C}$ \\
2 & $1 \mathrm{~g}: 0.7 \mathrm{~mL}$ & $0-5{ }^{\circ} \mathrm{C}, 20^{\circ} \mathrm{C}$ \\
3 & $1 \mathrm{~g}: 1.5 \mathrm{~mL}$ & $0-5{ }^{\circ} \mathrm{C}$ \\
4 & $1 \mathrm{~g}: 1.5 \mathrm{~mL}$ & $0-5{ }^{\circ} \mathrm{C}, 20^{\circ} \mathrm{C}$ \\
\hline
\end{tabular}

Scheme-II: In this design,vigorous reaction conditions including reaction media, reaction temperature, different ratio 


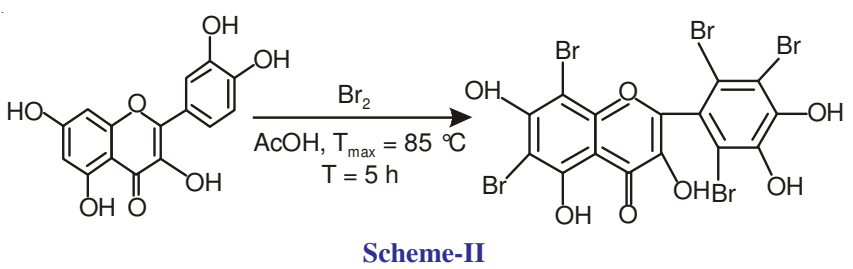

of starting materials and reaction time were studied. At the end, we found that glacial acetic acid as solvent, ratio of starting materials: quercetin: $\mathrm{Br}_{2}=1: 8$ (molar ratio), $\mathrm{T}_{\max }=85^{\circ} \mathrm{C}$ and $5 \mathrm{~h}$ reaction time are optimized reaction conditions to obtain final product $6,8,2^{\prime}, 5^{\prime}, 6^{\prime}$-pentabrominated quercetin. Interestingly, under these conditions, single component, $6,8,2^{\prime}, 5^{\prime}, 6^{\prime}-$ pentabrominated quercetin was obtained with simple purification and high yield (Table-2).

\section{TABLE-2}

REACTION OF QUERCETIN WITH $\mathrm{Br}_{2}$ IN THE FOLLOWING DIFFERENT CONDITIONS AND THE EXPLORATION RESULT

\begin{tabular}{cccc}
\hline Entry & Solvent & $\begin{array}{c}\text { Molar ratio of material } \\
\text { Quercetin: } \mathrm{Br}_{2}\end{array}$ & $\begin{array}{c}\text { Reaction time } \\
\left(\mathrm{T}_{\max }\right)\end{array}$ \\
\hline 1 & $\mathrm{CH}_{3} \mathrm{CH}_{2} \mathrm{OH}$ & $1: 4$ & $25^{\circ} \mathrm{C}$ \\
2 & $\mathrm{AcOH}$ & $1: 4$ & $25^{\circ} \mathrm{C}$ \\
3 & $\mathrm{AcOH}$ & $1: 4$ & $55^{\circ} \mathrm{C}$ \\
4 & $\mathrm{AcOH}$ & $1: 4$ & $85^{\circ} \mathrm{C}$ \\
5 & $\mathrm{AcOH}$ & $1: 8$ & $25^{\circ} \mathrm{C}$ \\
6 & $\mathrm{AcOH}$ & $1: 8$ & $55^{\circ} \mathrm{C}$ \\
7 & $\mathrm{AcOH}$ & $1: 8$ & $85^{\circ} \mathrm{C}$ \\
\hline
\end{tabular}

The NMR spectra of quercetin had five groups of peaks at approximately $\delta 6$ to $\delta 8$ for the five hydrogens on the benzene rings of quercetin. Hydrogen peaks between $\delta 6$ and $\delta 8$, of NMR spectra for product disappeared meaning that the product is $6,8,2^{\prime}, 5^{\prime}, 6^{\prime}$-pentabrominated quercetin. Its mass spectra indicated its molecular mass was 696.4 and had the correct ratio characteristic of fragments containing five bromine atoms.

\section{Conclusion}

In summary, two efficient bromination methodologies have been developed for modifying quercetin using $\mathrm{HBr}$ and $\mathrm{Br}_{2}$ under different chemical conditions. In general, the product yields are high and work-up is straightforward.

\section{ACKNOWLEDGEMENTS}

This work is supported by Initial Xiaoxiang Endowed Professorship grant provided by Hunan Normal University. The authors thank Dr. Changling Liu, CTO from Shenyang Research Institute of Chemical Industry for his constructive designs and creative discussions.

\section{REFERENCES}

1. M.A. Baig, V.B. Gawali, R.R. Patil and S.R. Naik, J. Nat. Med., 66, 500 (2012)

2. S.L. Pinto, J. Kumar, G. Partha and R.A. Bechtol, Clinicoecon Outcomes Res.., 5, 153 (2013).

3. L. Cialdella-Kam, D.C. Nieman, W. Sha, M.P. Meaney, A.M. Knab and R.A. Shanely, Br. J. Nutr., 109, 1923 (2012).

4. F. Boers, G. Lemiere, J.A. Lepoivre, A. De Groot, R. Dommisse, T. De Bruyne, A.J. Vlietinck and D.A. Vanden Berghe, Pharmazie, 53, 512 (1998).

5. C. Hu, Z. Jian-ping and H. Shao, The Synthesis Research of Quercetin Derivatives, Medicinal Chemistry, National Conference Proceedings (China), p. 159 (2005).

6. Yong-Sheng Dai, Master's Degree Thesis, The Structural Design and Synthesis of Flavonoid Derivatives, Harbin Engineering University, (2006).

7. J. Tan, W. Bo-Chu and Z. Lian-Cai, Chinese Pharm. J., 41, 1688 (2006).

8. B. Zhao, Q.H. Xu and L.Y. Duan, Chemical Reagents, 28, 141 (2006). (J).

9. M. Ichikawa, S. Hibino, M. Onishi, J.F. Hatcher, A.M. Pamukcu and G.T. Bryan, Org. Prep. Proced. Int., 17, 56 (1985).

10. V.V. Polyakov, Chem. Nat. Comp., 35, 21 (1999). 